\title{
Esthetic and functional rehabilitation in a case of amelogenesis imperfecta
}

\author{
Ramneet Kaur $^{1 *}$, Asha Karadwal ${ }^{2}$, Deepak Sharma ${ }^{3}$, Akhil Sharma ${ }^{4}$, Anirudh Pathak ${ }^{5}$ \\ ${ }^{1,2,5}$ Senior Lecturer, ${ }^{3}$ Former Professor, ${ }^{4}$ Reader, ${ }^{1,3-5}$ Dept. of Pediatric and Preventive Dentistry, ${ }^{2}$ Dept. of Oral Patholgy and \\ Microbiology, ${ }^{1,4}$ Yamuna Institute of Dental Sciences and Research, Haryana, ${ }^{2,3}$ M.M. College of Dental Sciences and \\ Research, Maharishi Markandeshwar University, Mullana, Ambala, Haryana, ${ }^{5}$ Himachl Dental College, Sundernagar, \\ Himachal, India
}

\section{Corresponding Author: Ramneet Kaur}

Email: ramneet37@gmail.com

\begin{abstract}
Amelogenesis imperfecta (AI) is a group of hereditary conditions that affects the quality and quantity of dental enamel that is occasionally associated with other general conditions. ${ }^{1}$ Patients with AI manifests increased dental sensitivity resulting in poor oral hygiene and compromised masticatory functions. Correct diagnosis and management is highly needed to restore the quality of oral health. We presented here a case of full mouth rehabilitation in a 7 year child with Amelogenesis Imperfecta.
\end{abstract}

Keywords: Amelogenesis Imperfecta, Enamel hypoplasia, Enamel hypomaturation.

\section{Introduction}

Phenotypically, AI may vary from mild discoloration to post eruptive enamel breakdown depending upon the stage of enamel matrix formation and maturation disturbed. During enamelogenesis, due to genetic mutation or chromosomal decfects in specific enamel proteins such as Enamelin gene (ENAM), Amelogenin gene (AMELX), Kallikrein 4 gene (KLK4), Matrix Metalloproteinase 20 gene (MMP-20), and Distal-less homeobox 3 gene (DLX3), resulting in Amelogenesis Imperfecta. $^{2-5}$ AI shows autosomal dominant, autosomal recessive, X-linked and sporadic inheritance patterns. It usually affects both primary and permanent dentitions.

\section{Case Report}

A 7 year old female child reported to the department of Pediatric and Preventive Dentistry with chief complaint of yellowish discoloration in upper and lower front teeth along with increased sensitivity to hot and cold from past 1 year. She also had difficulty in brushing her teeth. A detailed medical and family history was taken. Patient's mother had a similar kind of dental anomaly. There was no history of past dental treatment.

Intraorally, soft tissue examination revealed no abnormality. Patient had mixed dentition with permanent incisors $(11,21,12,31,32,41,42)$ showing chalky white appearance with localized yellowish discoloration due to chipping of enamel at the incisal edges exposing underlying dentin [Figure 1a]. Mottled, opaque- white facial surface with thinning of enamel towards occlusal surfaces exhibiting marked yellowish brown discoloration on deciduous $1^{\text {st }}$ and $2^{\text {nd }}$ molars $(54,55,64,65,74,75,84$, 85) of both arches was observed [Figure 1b,c and Fig. $2 \mathrm{a}, \mathrm{b}]$. Clinical height of deciduous $1^{\text {st }}$ molars was also reduced. Proximal dental caries were seen in 74, 75 [Fig. 2b]. Deciduous canine revealed yellowish discoloration of enamel. Mandibular $1^{\text {st }}$ permanent molars revealed thin mottled enamel with absence of enamel at certain points on occlusal surface [Fig. 2b]. Panoramic radiograph revealed thin layer of enamel on deciduous molars and canines with less contrast between enamel and dentin. It also showed absence of enamel at incisal edges and occlusal surfaces of permanent incisors and molars [Fig. 3]. On the basis of clinical and radiographic features, final diagnosis of AI - Hypomaturation, Hypoplastic type was confirmed.

Treatment protocol included preventive, functional and esthetic rehabilitation. Since the patient's diet history revealed high cariogenic diet, preventive care involved oral nutritional counseling and oral health care instructions along with the use of fluoridated mouthwash. Topical fluoride application sessions were scheduled twice a year. Oral prophylaxis was carried out. Pit and fissure sealants were placed on 16, 26 [Fig. 5a]. Increased tooth sensitivity added to the challenge in repairing the teeth. Stainless steel crowns were placed on $54,64,65,74,75,84,85$ to restore vertical clinical dimensions and to protect the teeth from further breakdown [Fig. 4b,c and Figure 5a,b]. Finally, the direct composite restorations were executed in 36, 46 [Fig. 5b] and on 11, 12, 21, 31, 41, 32,42 for esthetic correction that also alleviated pain and sensitivity [Fig. 4a]. 


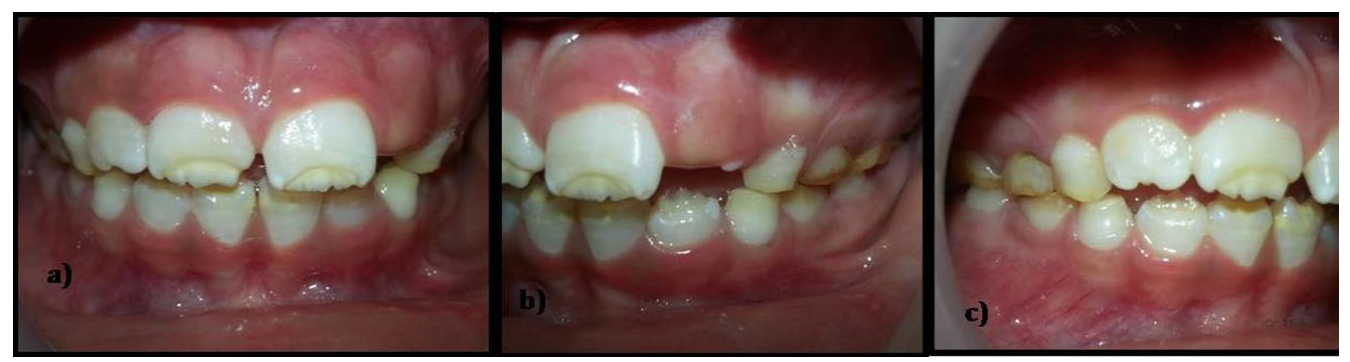

Fig. 1: a) Frontal view in occlusion revealing chipped enamel in permanent incisors b,c) Right \& left lateral view in occlusion respectively.

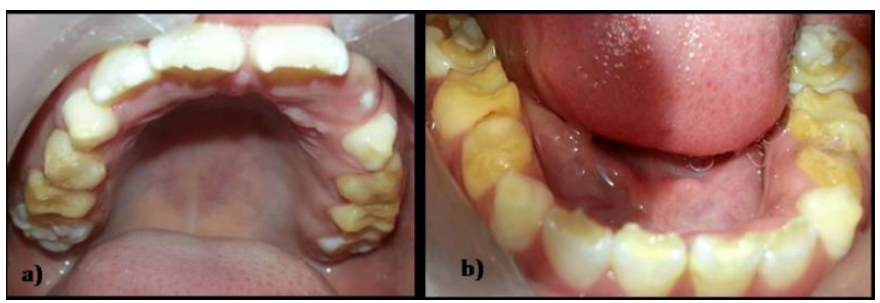

Fig. 2: a) Intraoral view of maxillary arch showing presence of mottled enamel b) Intraoral view of mandibular arch revealing mottled enamel on occlusal surfaces and proximal caries in 74,75 .

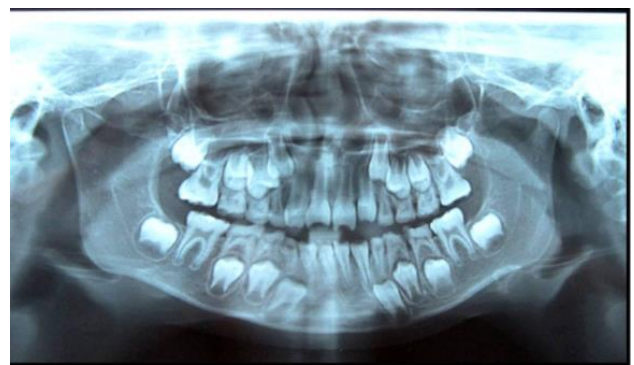

Fig. 3: Orthopantomogram revealed less contrast between enamel and with loss of enamel at occlusal surface and incisal edges..

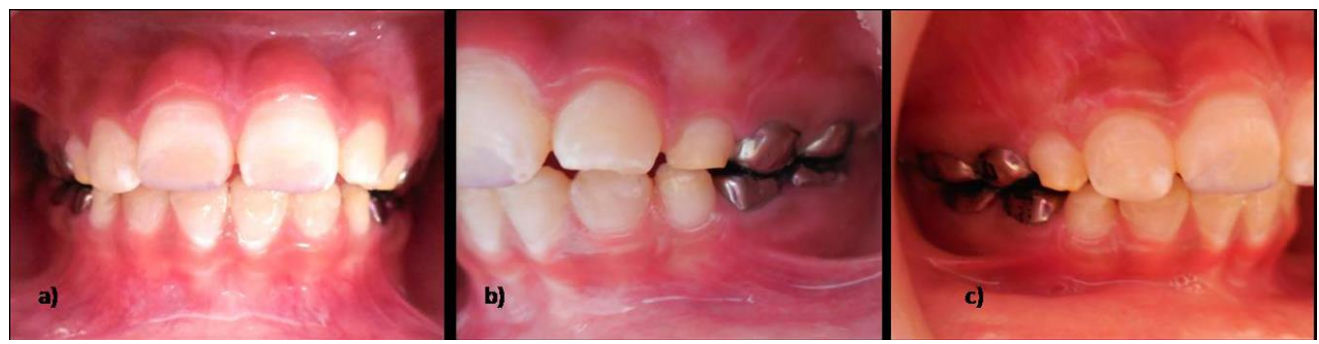

Fig. 4: a) Esthetic correction done conservatively by composite restrations in 11, 21, 12, 31, 32. b,c) Stainless steel crowns on deciduous molars restored clinical vertical heights of crown.

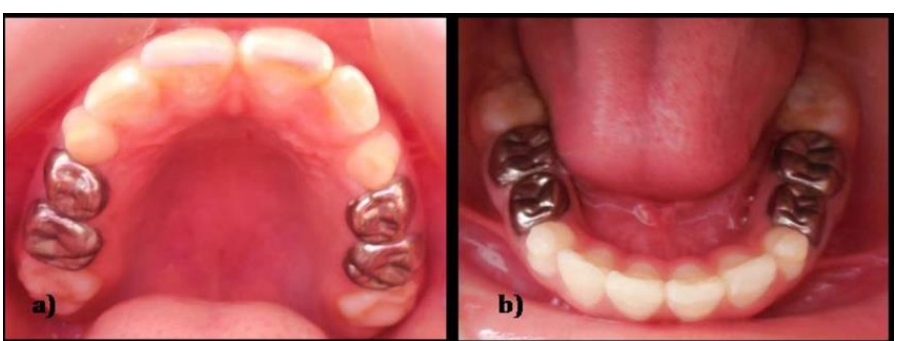

Fig. 5: a) Pit and fissure sealants done on 16, 26 and stainless steel crowns placed on maxillary deciduous molars. b) Composite restorations done on 36, 46 and stainless steel crowns placed on mandibular deciduous molars. 


\section{Discussion}

According to Wiktop's classification, the defects of AI are primarily classified based on their phentotypes as hypocalcified (soft enamel that can be easily removed), hypomaturation (chalky-opaque enamel with chipping of enamel), hypoplastic type (reduced quantity of enamel) and hypoplastic-hypomaturation with taurodontism types. ${ }^{6}$

Dental fluorosis, Molar Incisor Hypomineralization (MIH), chronological and localized disorders of tooth formation should also be considered in the differential diagnosis. Most common differential diagnosis is dental fluorosis, that may vary from mild white "flecking" to confluent pitting of enamel but it's usually associated with history of high fluoride intake. ${ }^{7}$ Chronological disorders that may arise from any cause such as celiac disease etc. during tooth formation may also lead to mottled and hypoplastic enamel. MIH is decribed as the hypomineralization of systemic origin that affects one to four first permanent molars, frequently associated with affected permanent incisors. ${ }^{8}$ In the presented case, all the teeth including primary and permanent dentition were affected with no systemic association. Also, the presence of family history supported the clinical and radiographical findings and lead to the confirmation of final diagnosis.

AI presents as a challenge to the clinicians as the post eruptive enamel breakdown usually occurs shortly after their eruption leading to the hypersensitivity, rapid caries progression which demands the use of anesthesia even while doing restorations. Patient compliance is needed the most as the number of appointments increases. Patience and behaviour management are important tools to handle a young and fearful child. Patients mainly complains of unaesthetic appearance, dental sensitivity, ectopic eruption, congenitally missing teeth, early or late eruption of teeth, difficulty in brushing and difficulty in maintaining hygiene. ${ }^{9,10}$ The children with AI demonstrated emotional and physical strains that affected in maintaining long term relationships as compared to other children. ${ }^{11}$ Esthetic correction and reduced dental sensitivity in treated teeth helps in improved masticatory function and motivates the child patient to complete the treatment.

Treatment protocol involves Preventive, functional and esthetic correction. ${ }^{12}$ Preventive approach that includes diet counseling and oral hygiene care at home including use of fluoridated toothpaste and mouthwash is recommended. Pit and fissure sealants and fluoride applications to prevent future lesions in the deep pits are performed as preventive measures in clinic. In primary dentition, the molars are protected by preformed stainless steel crowns. Restorative aspects includes composite restorations, polycarbonate crowns and zirconia crowns depending upon the severity of the defect. $^{13}$. Minimally invasive procedure was excecuted in this case to repair chipped incisal edges by composite restorations in anterior teeth.

\section{Conclusion}

Early and correct diagnosis of AI aids in implementing preventive measures and correct restorative techniques. Thus, avoiding the necessity of complicated and invasive treatment in future. Esthetic correction affects positively on the psychology of the patient thereby improving their social behavior.

\section{Source of funding}

None.

\section{Conflict of interest}

None.

\section{References}

1. Witkop CJ. "Hereditary defects in enamel and dentin". Acta Genet Stat Med 1957;7(1):236-9.

2. Wright JT, Torain M, Long K, Seow K, Crawford $\mathrm{P}$, Aldred MJ et al, "Amelogenesis imperfecta: Genotypephenotype studies in 71 families". Cells Tissues Organs 2011;194(2-4):279-83.

3. Kim JW, Seymen F, Lin BP, Kiziltan B, Gencay K, Simmer JP et al, "ENAM mutations in autosomal-dominant amelogenesis imperfect". J Dent Res 2005;84:278-82.

4. Hu JC, Simmer JP. "Developmental biology and genetics of dental malformations". Orthod Craniofac Res 2007;10(2):45-52.

5. Crawford PJ, Aldred MJ. "Clinical features of a family with $\mathrm{X}$ linked amelogenesis imperfecta mapping to a new locus (AIH3) on the long arm of the X chromosome". Oral Surg Oral Med Oral Pathol 1993;76:187-91.

6. Witkop CJ. “Amelogenesis imperfecta, dentinogenesis imperfecta and dentin dysplasia revisited: Problems in classi-fication". J Oral Pathol 1988;17(9-10):547-53.

7. Majumdar KK. "Health impact of supplying safe drinking water containing fluoride below permissible level on flourosis patients in a fluoride-endemic rural area of West Bengal”. Indian J Public Health 2011;55(4):303-8.

8. Weerheijm K L, Jalevik B, Alaluusua S. "Molar-Incisor Hypomineralisation". Caries Res 2001;35(5):390-39.

9. Seow W. "Dental development in amelogenesis imperfecta: A controlled study". Pediatr Dent 1995;17(1):26-30.

10. Poulsen S, Gjorup H, Haubek D. "Amelogenesis imperfecta - A systematic literature review of associated dental and oro-facial abnormalities and their impact on patients". Acta Odontol Scand 2008;66(4):193-9.

11. Coffield K, Philips C, Brady M, Roberts M, Strauss R, Wright JT et al. "The psychosocial impact of developmental dental defects in people with hereditary amelogenesis imperfecta". J Am Dent Assoc 2005;136(5):620-30. 
12. American Academy of Pediatric Dentistry. "Guideline on Dental Management of Heritable Dental Developmental Anomalies". Pediatr Dent 2016;38(6):302-7.

13. Turkün L. "Conservative restoration with resin composites of a case of amelogenesis imperfecta". Int Dent J 2005;55(1):38-41.
How to cite: Kaur R, Karadwal A, Sharma D, Sharma A, Pathak A. Esthetic and functional rehabilitation in a case of amelogenesis imperfect. Int Dent J Student Res 2020;8(1):33-6. 